\title{
A CONSTRAINT-BASED APPROACH TO TRANSLATING ANAPHORIC DEPENDENCIES
}

\author{
Louisa Sadler \\ Doug Arnold \\ Department of Language \& Linguistics, \\ University of Essex, Wivenhoe Park, \\ Colchester, CO4 3SQ, UK \\ (louisa@essex.ac.uk;doug@essex.ac.uk)
}

\begin{abstract}
The normal method for representing anaphoric dependencies in Unification Based grammar formalisms is that of re-entrance. In this paper, we address the problems that this representational device poses when such formalisms are used for translation. We demonstrate the inadequacies of existing proposals, and describe an approach which exploits the expressive possibilities of the equational constraint language in LFG and involves an inferential procedure combining underspecification in the statement of bilingual correspondences with the use of target language knowledge.
\end{abstract}

\section{Introduction}

The normal method for representing anaphoric dependencies in Unification Based (UB) grammar formalisns is that of re-entrance (often indicated graphically by means of subscripts, as in (1b)). The interpretation is that two or more attributes share (are pointers to) a sin. gle value. In the case of (1), the SUBJ of try is identical to that of employ. This means that changes to the value of the SUBJ of try (c.g. the addition of another feature) are necessarily changes to the value of the SUBJ of employ. This token identity should be distinguished from the type identity between these values, and the value of the $O B J$ attribute in (1b), which just happens to have the same attributes and values.

\section{(1a) Linguists try to employ linguists.}

This device can be thought of as the attributevalue equivalent of a bound variable of logic, and though it is not appropriate for all kinds of anaphoric dependence, it is ubiquitous.

This paper proposes a novel approach to the treatment of re-entrances in translation, which overcomes the problems of existing approaches using UB formalisms. These prob- (1b)

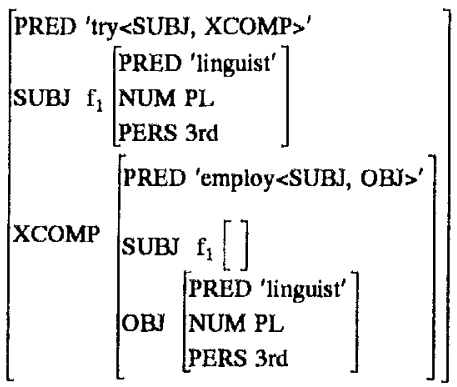

lems are considerable, but have been generally ignored in the literature. Section 2 will review existing approaches, pointing out the problems. Section 3 describes the approach, which provides a straightforward treatment of cases where filler-gap dependencies are subject to different constraints in source and target languages (c.g. where one language allows, and the other forbids, Preposition Stranding). In exemplifying this, we will focus on the treatment of relative clauses. ${ }^{1}$

\section{Existing Approuches}

\subsection{Transfer Based (Structural) Approaches.}

Perhaps the most obvious way to use a UB formalism for translation is to use a standard UB grammar formalism in analysis to produce a feature structure (FS), which is a collection of attribute-value (A:V) pairs. This is then mapped to a target FS by means of a bilingual grammar of transfer rules whose left-hand-sides (lhs) are matched against the source structure,

\footnotetext{
1 Elsewhore, we discuss casos where fller-gap dependencies in one langurge correspond to antecedent-pronoun dependencios in another.
} 
and whose right-hand-sides (rhs) indicate the content of the corresponding target FS. These rules are applied recursively to successively smaller collections of source language $A: V s$. This is, of course, just a straightforward adaptation of the traditional transfer method to a $A: V$ data structure in place of a tree, in particular, it resembles a classical transter system in being "structural" i.c. in involving the decomposition of source structures into smaller objects (on the Ihs), and the actual construction of target structures (on the rhs). This is essentially the approach employed in ELU (Estival et al 1990) and MiMo2 (van Noord et al 1990).

Though there is not much discussion of the treatment of referential dependencies in transfer in these formalisms, it is easy to see how one can deal with re-entrances which naturally fall within the scope of one transfer rule. In ELU, for example, such re-entrances can be translated by binding the re-entrant paths within the structure to the same variable and stating a correspondence between the relevant source side and the target side variables. In this way the re-entrance is translated as one structure. In MiMo2 the re-entrant paths are separately translated, but the re-entrance is explicitly mentioned on source and target side, requiring token-identity between the results of the separate translations. However, these structure based formalisms do not have any method for generalizing this to cases where re-entrances are not 'local'. This is serious, because phenomena classically regarded as involving Wh-Movement (e.g. Wh-Questions, Topicalization, Relativisation, etc.), are typically of this kind, and for these phenomena, the formalisms can provide no general treatment.

Of course, there are a number of ways in which one might try to remedy this inadequacy. For example, one could unfold the re-entrances as type identities (i.e. reinterpret the DAG as a tree), or 'thread' shared values through the structure, in such a way that they become local (cf. standard gap threading techniques to reduce unbounded dependencies to local ones). However, none are satisfactory. The former looses information, so that source FS and target FS are no longer equivalent, and causes problems in generation, where some method must be found for ensuring that lexical content is not duplicated, and appears in the right place). Threading techniques are unattractive because of the (often extreme) complication they introduce in gram- mars and representations. ${ }^{2}$

\subsection{Constraint Based Approaches}

In this section, we will outline the approach to the translation of non-local reentrances proposed in Kaplan et al (1989).

In LFG projections are linguistically relevant mappings or correspondences between levels, whether these mappings are direct or involve function composition (Kaplan (1987), Halvorsen and Kaplan (1988), Dalrymple (1990) and Dalrymple et al (1990)). By means of these projections, equations can be stated which codescribe clements of the two levels related by the projection. The standard projections are $\psi$ (normally expressed in terms of $\uparrow$ and $\downarrow$, from c-structure to f-structure), and $\sigma$ (variously from c- and f-structure to semantic structures). Kaplan et al extend this approach to provide what amounts to a transfer formalism for LFG. ${ }^{3}$ In their proposal, the equational language of LFG is used to state bilingual constraints or correspondences between glements of source and target structures. They introduce mapping functions $\tau$ (between f-structures) and $\boldsymbol{x}^{\prime}$ (between semantic structures). Achieving translation can be thought of as specifying and resolving a set of constraints on target structures, constraints which are expressed by means of the $\boldsymbol{r}$ and $\boldsymbol{r}^{\prime}$ functions.

The formalism permits a wide variety of source-target correspondences to be expressed: $t$ and $\psi$ can be composed, as can $\tau^{\prime}$ and o. Equations specifying translations are added to (source language) lexical entries and c-structure rules. For example (2) composes $\tau$ and $\psi$, identifying the $\tau$ of the (source) SUBJ f-structure with the SUBJ attribute of the $\tau$ of the f-structure associated with some node (the value of $\uparrow$ ), indicating that the translation of the value of the SUBJ slot in a source t-structure fills the SUBJ slot in the f-structure which is the translation of that source

\footnotetext{
2 The poosibility of a 'threasling' approach is hintod at in van Noord of $\alpha$ (1990). The formalisan described in Pulman (ed) (1991) seems to allow an interesting vatiation, where instend of throeding information about nou-jocal re-entrance through the source atructure, it is threaded through the "virtual' atructures that are built as the transfer mechanisms recurses through the source structure. This still does not avoid the bavic objection to the use of such techriques, however.

3 Soe Sadler at al (1990), Sadler and Thompoon (1991), and Sadler (1991) for further discussion of this spproach to MT.
} 
f-structure.

(2) $\tau(\uparrow S U B J)=(\tau \uparrow$ SUBJ $)$

In this approach, then, relations between different types of linguistic description (i.e. levels) are defined in terms of correspondence functions, not by means of the recursive application of rules to source language structures. In particular, notice that transfer does not operate compositionally on a source language feature structure, rather the analysis procedure collects sets of constraints on various structures, including $(\tau)$ constraints on target structures. The solution of a set of $\tau$ equations is a (probably incomplete) target f-structure which must then be completed and validated by the target grammar. This allows information which is exhaustively determined by the target grammar to be ignored in the transfer process. ${ }^{4}$ In this sense, the system is constraint-based, rather than structure based like the approaches described in 2.1. above, and it has different expressive possibilities.

As regards relative clauses, Kaplan et al assume a reasonably standard LFG analysis: wh-relatives are represented as an attribute (here RELMOD) which contains a re-entrance between the values of a RELTOPIC phrase and a within-clause function (see (8) below). The approach to translating these dependencies involves stating separate correspondences for both the within clause function and the RELTOPIC function.

For a simple example like (3), with English as source language, the rules are as in (4-7) and the English and (incomplete) French f-structures as in (8) and (9) (the indices here are simply informal devices to allow easy reference to pieces of $f$-structure).

(3)a. The man who I saw.

b. L'homme que j'ai vu.

\footnotetext{
4 For a turgot astitenco to be trandation of a cource entence, the minimal structure aseigned to the target sentence by the unget grammer mum be subsumed by the minimal solution of the $\tau$ and $\tau^{\prime}$ con. anint.
}

(4)

NP

$\rightarrow \mathrm{NP}$

$$
\begin{gathered}
\text { S' }^{\prime} \\
\uparrow \text { RELMOD }-\downarrow \\
\tau(\uparrow \text { RELMOD })=(\tau \uparrow \text { RELMOD }) \\
\tau(\downarrow \text { RELTOPIC })=(\tau \downarrow \text { RELTOPIC })
\end{gathered}
$$

(5)

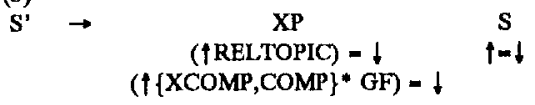

(6) see: $V$

PRED r'see<SUBJ,OBJ>'

$\tau(\uparrow$ SUBJ $)-(\tau \uparrow$ SUBJ $)$

$\tau(\uparrow O B J)-(\tau \dagger \circ B)$

(T) who: $\mathrm{N}$

PRED='who'

HUMANm+

( $\tau \uparrow$ PRED FN) - QUE'6 $^{\prime 6}$

In the functional uncertainty equation in (5), $\{\mathrm{XCOMP}, \mathrm{COMP}\}$ * allows the 'gap' associated with the RELTOPIC to be inside zero or more COMPs or XCOMPs, and GF is an abbreviation for a set of paths including length one paths such as SUBJ, OBJ, etc., and paths of length two, such $\mathrm{OBL}_{20} \mathrm{OBJ}$, which allows preposition stranding, as in man who I replied to []$_{\text {i }}$

(8)

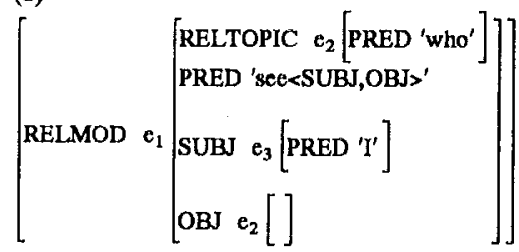

(9)

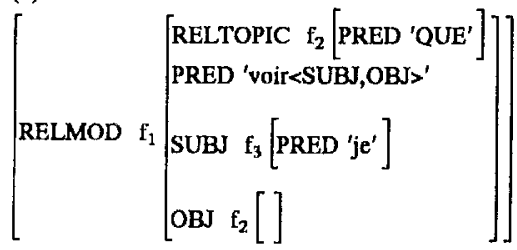

The equations on rule (4), which are specifically for dealing with relative structures, are quite simple in themselves, and combine

\footnotetext{
- Wo assume that PRED='QUE' subaumes the varients que, qui, baqualle, etc.
} 
with the equations given in the lexical entries (6) and ( $T$ ) to create a re-entrance in the target structure (9) corresponding to that in (8). This can be seen by looking at the relevant constraints, in (10), which are derived from these rules in relation to $(8)$ and $(9)$. Since $\tau$ is a function, ( $f_{1}$ RELTOPIC) and ( $f_{1} O B J$ ) must be the same token. Hence the desired reentrance falls out automatically.

$$
\begin{aligned}
& \left(f_{1} \text { RELTOPIC }\right)-\tau\left(e_{1} \text { RELTOPIC }\right)-\tau\left(c_{2}\right) \\
& \left(f_{1} \text { OBJ }\right)-\tau\left(e_{1} \text { OBJ }\right)-\tau\left(c_{2}\right)
\end{aligned}
$$

Thus, it appears that, in principle, this approach requires neither the addition of special apparatus, nor modifications to the ireatment of grammatical phenomena that do not involve teentrance.

Unfortunately, this approach is only capable of producing intuitively correct structures in cases where the conditions on unbounded dependencies are parallel in the source and target languages. On closer inspection, the example Kaplan et al use to demonstrate their approach does not work correctly, giving the ungrammatical (11c) instead of the correct (11b).

(11)a The letter which I have answered.

b. La lettre à laquelle j'ai répondu. The letter to which I have responded

c. La lettre laquelle j'ai répondu à. The letter which I have responded to

The c-structure rules and annotations required here include (4) and (5), and the lexical entry for answer, which includes the information in (12). The source f-structure produced by these rules is (13).

$$
\begin{aligned}
& \text { †PRED='answer<SUBJ, OBJ>' } \\
& \text { ( } \tau \uparrow \text { PRED FN)-'repondre }<S U B J, O^{\circ} L_{80}>\text { ' } \\
& \tau(\uparrow \text { SUBJ })-(\tau\{\text { SUBJ }) \\
& \tau(\uparrow \circ B)-\left(\tau \nmid \mathrm{OBL}_{\mathrm{go}} \text { OBJ }\right)
\end{aligned}
$$

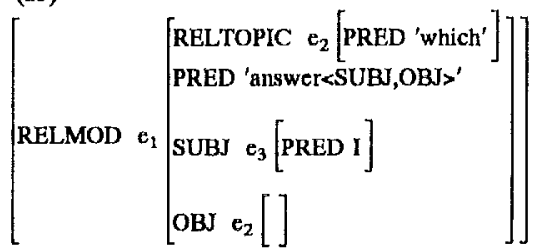

From these rules, the following $\tau$ equations arise, in relation to (13):
(14)

$$
\begin{aligned}
& \tau(\uparrow \text { RELTOPIC })-(\tau \uparrow \text { RELTOPIC }) \\
& \tau\left(e_{2}\right)-\left(f_{1} \text { RELTOPIC }\right) \\
& \tau(\uparrow O B J)=\left(\tau \uparrow \text { OBL }_{\mathrm{go}} \text { OBJ }\right) \\
& \tau\left(e_{2}\right)=\left(f_{1} O B L_{\mathrm{go}} \text { OBJ }\right)
\end{aligned}
$$

However, these yield the incorrect f-structure (15) (if we assume details filled in from the monolingual grammar), corresponding to the ungrammatical string (11c), with a stranded preposition. $^{7}$

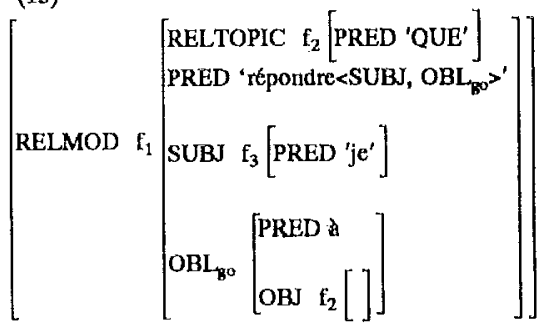

The problem in this case arises because the relativised positions in English and French are not identical. Although it seems at first sight that the approach nicely preserves reentrances in translation, in fact what happens is that the source grammar dictates what will be re-entrant on the target side. Thus, though the Kaplan et al approach provides a simple method for projecting source language re-entrances onto the target language structures, the method is insufficiently liexible in the scope allowed for variation between source and target structures.

\section{Using Underspecification}

Characteristic of the approaches described in 2.1. and 2.2. is that they translate both 'ends' of a re-entrance. Because in structure based systems the translation relation is defined entirely by rules, the scope of the re-entrances that can be handled is limited by that of rules. A constraint based approach avoids this problem -. under the approach described in 2.2 separate $x$ correspondences are supplied for both paths involved in the re-entrance (both the source RELTOPIC, and the source within-clause function). Because these correspondences apply $\tau$ to f-structure descriptions which evaluate to the same object, a target re-entrance is automati-

\footnotetext{
7 This representation embodies an number of questionable assumptions sbout the treatment of a which are not relevant to the discussion.
} 
cally established, whose value is the translation of this object. However, as we have seen, this approach does not permit factorization of source and target oriented information in these cases. In this section we will explore a solution to this problem which involves restricting $\tau$ correspondences to just one of the paths involved in the source re-entrance, allowing a constraint based treatment of cases including those in (11) above. ${ }^{8}$ This possibility is not easily available in 'structure based' approaches, and represents a genuine advantage of a constraint based approach.

\subsection{Different Ke-entrances in Source and Target}

Suppose that no $\tau$ equations are stated on the c-structure rules introducing the RELTOPIC attribute, and a $\tau$ correspondence is stated only over the path terminating in the within clause (thematic) function. What results would be a French f-structure like (16), which differs from (15) only in the absence of a RELTOPIC, and would correspond to the string $j$ 'ai répondu à laquelle ('I have responded to which'): (16)

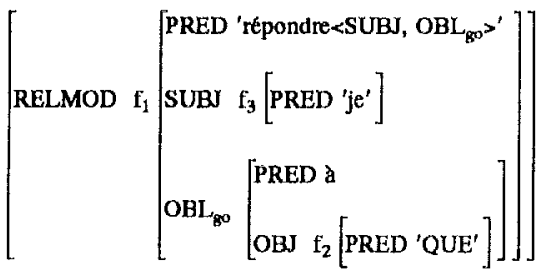

In order to produce an f-structure corresponding to (11b), i.e. a translation of (11a), we must ensure that an appropriate value for a RELTOPIC attribute is given. There are three sources of potentially useful information here.

First, there is some source-oriented information - the solution to the functional uncer-

\footnotetext{
B Kaplen et al propose just auch a treatment in cases litwe the translation of John is likely to see Mary -Il art probable que Jean verra Marie. No $\tau$ comespondence is given for the SUBJ of likely, so the f-structure associatod with John is only related to a targot f-structure in the thematic position (SUBJ of soe). The French monolingual loxioon supplies an axpletive SUBJ for probable. Howover, Keplan at al do not consider the possibility of dealing with 'unbounded' re-entrances in this way.
}

tainty equation associated with the XP node in the English c-structure. The solution of the functional uncertainty equation in this case happens to be ( $\uparrow$ RELTOPIC)-( $\uparrow$ OBJ). By the application of a general schema, we can derive a $\tau$ equation from this, which in this case is (17), which (again in this case) is equivalent to (18).

$$
\begin{aligned}
& (\tau \uparrow \text { RELTOPIC })-\tau(\uparrow \text { OBJ }) \\
& (\tau \uparrow \text { RELTOPIC })-\left(\tau \uparrow \mathrm{OBL}_{\mathrm{g}} \text { OBJ }\right)
\end{aligned}
$$

The method for doing this involves taking the functional uncertainty, namely † RELTOPIC = $\uparrow\{\text { XCOMP,COMP }\}^{*} \mathrm{GF}$, and adding ( $\uparrow \uparrow \mathrm{REL}$ TOPIC $)-\tau(\uparrow \alpha)$ for every solution $\alpha$ of the uncertainty on the right-hand side. This gives (18) as one solution.

Of course, this cannot simply be added to the other $x$ equations (if it were, it would establish a re-entrance between RELTOPIC and $\mathrm{OBL}_{\mathrm{g}}$ OBJ, which would give an ungrammati. cal result, with a stranded preposition, as in (11c) above).

Second, the monolingual target grammar will contain a constraint to ensure that, if RELTOPIC is present, some path within the RELTOPIC attribute contains the attribute value pair WHe+. This is required to prevent the 'topicaljzing' (i.e. wh-movement fronting within the relative clause) of any XP which does not contain a wh-phrase. Simplifying slightly, we take this equation to be:

$$
\text { ( } 1 \text { RELTOPIC }\{\text { OBJ, POSS }\} * \text { WH) } m_{c}+
$$

Third, the target grammar itself contains a functional uncertainty equation for establishing a relation between RELTOPIC and some within-clause function, which, for the sake of argument we could assume to be as in (20). Notice that this is more restrictive than the corresponding English constraint, which allowed identity between the values of a wide variety of GFs and the RELTOPIC. This restricts it to SUBJ, OBJ, and 'thematic' OBLiques (which includes $O B L_{g o}$ ), excluding the possibility of preposition stranding.

$$
\begin{gathered}
(\uparrow \text { RELTOPIC) }= \\
\left(\uparrow\{\text { COMP, XCOMP }\} *\left\{\text { SUBJ, OBJ, OBL }{ }^{2}\right\}\right)
\end{gathered}
$$

Intuitively, the source-derived equation (18) is used to provide the information that there should be a RELTOPIC attribute in the 
target f-structure. It can be interpreted defeasibly in combination with the target information to find the closest possible solution consistent with the target grammar. This closest solution cmerges from comparing the constraint with the functional uncertainty equations for the target language. In the case of relative clauses, at least, there are two target functional uncertainty equations - - the first expresses a re-entrance between the value of RELTOPIC and the value of some within clause function, and the second requires RELTOPIC to contain a WHe-t path ((1.9) and (20)).

If the source-derived equation is consistent with the target constraints, then it is chosen. If it is not, then the closest solution is chosen. Note that the shortest path in (19) would have just the wh-item in KELTOPIC (the OBJ of the preposition). But this is ruled out by $(20)$, which disallows $\mathrm{OBI} \mathrm{go}_{\mathrm{g}}$ OBJ as the bottom of the uncertainty path. "The "closest" solution is defined as the permissible solution which contains the minimal solution of the equation (19) (which requires RELTOPIC to contain a + WH item). In this casc, that solution is:

(21) († OBL $\left.\mathrm{OBO}_{\mathrm{go}}\right)=(\uparrow \mathrm{RELTOPIC})$

(22) († RELTOPIC OBJ WH) $=+$

In cobination with the other constraints, this will give a representation like (23), corresponding, to (11)b, as intended.

(23)

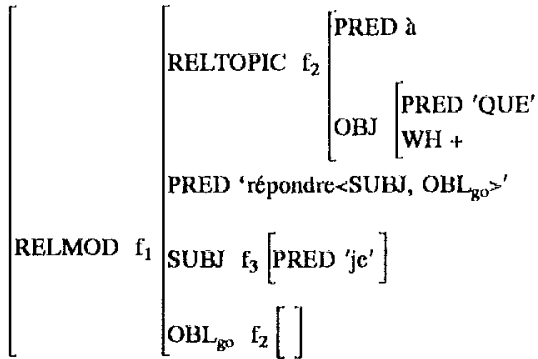

In this case, since French requires piedpiping rather than preposition stranding, the closest solution turns out to be the one which involves the attribute-value structure which contains the one specifled in the source-oriented constraint, with no other containing possible structure intervening. But the mechanism can be applied equally well to derive "smaller" RELTOPIC phrases from "larger" structures, as in the English $\rightarrow$ Irctich pair in (24), and can be extended to deal with 'strategy mismatches' of the kind exemplified in (25), where a 'gap' in one language corresponds to a resumptive pronoun in another (see Amold and Sadler 1992).
(24)a The man [ whose wife I have seen [];

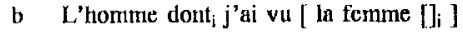 the man of-who I-have seen the wife
(25)a l'uomo che mi domando [ chi abbia visto I]
b the man of whom I wonder who he saw []

It is worth considering why this sort of method is not readily usable in "structure based" approaches. As here, the basic idea would be to translate the material in the within clause position only, ignoring the RELTOPIC position, and then create a re-entrance on the target side. 'There are at least two problems. First, in structural approaches, the nomal operation of transfer requires some kind of completeness chick to ensture that all parts of the source structure are translated. Nomally, this can be interpreted as a check that every path in the source object has been visited. Thus, for this approach to work, one would need rules that explicitly translate the value of RELTOPIC as nil ('deleting' it). One could, altentatively, try to redefine completeness in terms of translation of all values (since the RELTOPIC and the within clause position have the same value, translating either would count as translating both). This would mean one could avoid the rules explicitly deleting the RELTOPIC, but it is not clear what consequences it would have else. where. The second problem is nore serious. The output of transfer will produce a structure like man ( II I have seen who], and one will need rules to create a link between the RELTOPIC position ( $/ 7)$, and who. But one cannot, in general, assunie that such rules will exist. For examplc, thcy will not cxist if the turget grantmar creates links as part of the parsing process that creates A:V structures (e.g. if they anc associated with c-structure rules), and even if they are rules that can be applied to already constructed A:V structures, it cannot be guaranteed that they will apply to configurations such as this (since they will have been written to apply to cases where the lexical material (who) fconmands the 'gap'; but in the structures output from transter, the relationship will be the reverse. 


\section{Conclusion}

We have shown in this paper that the approach to transfer between feature structures introduced in Kaplan et al 1989 can be exploited to deal with the translation of anaphoric dependencies. Our proposal exploits the constraint based (rather than structure based) nature of the approach, and the flexibility that comes from being able to underspecify various parts of the translation relation, and allow information (i.e. constraints) from source language, and target grammar to interact with bilingual information.

\section{References}

(Abbreviations: 'TMI, International Conference on Theoretical and Methodological Issues in Machine Translation; EACL, Conference of the European Chapter of the Association for Computational Linguistics).

1. Doug Arnold and Louisa Sadler, "Empiricism, Rationalism, and Anaphoric Dependencies," Proceedings of TMI-4, Montreal, 1992.

2. Dominique Estival, Afzal Ballim, Graham Russell, and Susan Warwick, "A Syntax and Semantics for Feature-Structure Transfer," Proceedings of TMI-3, Linguistics Research Center, Austin, Texas, 11-13 June, 1990.

3. P-K. Halvorsen and R.M. Kaplan, "Projections and Semantic Description in Lexical-Functional Grammar," International Conference on Fifth Generation Computer Systems, Tokyo, Japan, 1988.

4. R. M. Kaplan and A. Zaenen, "LongDistance Dependencies, Constituent Structure, and Functional Uncertainty," in Alternative Conceptions of Phrase Structure, ed. M. R. Baltin and A. S. Kroch, pp. 17-42, Chicago University Press, Chicago, 1989.

5. Ronald Kaplan, Klaus Netter, Jurgen Wedekind, and Annic Zaenen, "Translation by Structural Correspondences," Proceedings of EACL-4, pp. 272-81, Manchester, 1989.

6. Ronald M. Kaplan, "Three seductions of computational psycholinguistics," in Linguistic Theory and Computer Applica- tions, ed. Peter Whitelock, Mary McGee Wood, Harold L. Somers, Rod L. Johnson and Paul Bennett, pp. 149-188, Academic Press, London, 1987.

7. S.G. Pulman (ed), EUROTRA ET6/1: Rule Formalism and Virtual Machine Design Study, Commission of the European Communities, Luxembourg, 1991.

8. Louisa Sadler, Ian Crookston, Doug Arnold, and Andy Way, "LFG and Translation," Proceedings of TMI-3, Linguistics Research Center, Austin, Texas, 11-13 June, 1990.

9. Louisa Sadler and Henry S. Thompson, "Structural Non-Correspondence in Translation," Proceedings of EACL-5, pp. 293. 8, Berlin, 1991.

10. Louisa Sadler, "Structural Transfer and Unification Formalisms," Applied Computer Translation, vol. 1 , no. 4, pp. 1-22, 1991.

11. Gertjan van Noord, Joke Dorrepaal, Pim van der Eijk, Maria Florenza, and Louis des Tombe, "The MiMo2 Research System," Proceedings of $T M I-3$, Linguistics Research Center, Austin, Texas, 11-13 June, 1990. 